\title{
SYSTÈMES D'INNOVATION ET COMMUNAUTÉS DE CONNAISSANCES DANS LE SECTEUR AGRICOLE ET AGROALIMENTAIRE
}

\author{
Jean-Marc Touzard et al. \\ De Boeck Supérieur | Innovations
}

\section{$2014 / 1-n^{\circ} 43$ \\ pages 13 à 38}

ISSN 1267-4982

Article disponible en ligne à l'adresse:

http://www.cairn.info/revue-innovations-2014-1-page-13.htm

Pour citer cet article :

Touzard Jean-Marc et al., « Systèmes d'innovation et communautés de connaissances dans le secteur agricole et agroalimentaire »,

Innovations, 2014/1 n 43, p. 13-38. DOI : 10.3917/inno.043.0013

Distribution électronique Cairn.info pour De Boeck Supérieur.

(c) De Boeck Supérieur. Tous droits réservés pour tous pays.

La reproduction ou représentation de cet article, notamment par photocopie, n'est autorisée que dans les limites des conditions générales d'utilisation du site ou, le cas échéant, des conditions générales de la licence souscrite par votre établissement. Toute autre reproduction ou représentation, en tout ou partie, sous quelque forme et de quelque manière que ce soit, est interdite sauf accord préalable et écrit de l'éditeur, en dehors des cas prévus par la législation en vigueur en France. II est précisé que son stockage dans une base de données est également interdit. 


\title{
SYSTĖMES D'INNOVATION ET COMMUNAUTÉS DE CONNAISSANCES DANS LE SECTEUR AGRICOLE ET AGROALIMENTAIRE
}

\author{
Jean-Marc TOUZARD \\ INRA, UMR Innovation, Montpellier \\ touzard@supagro.inra.fr \\ Ludovic TEMPLE \\ CIRAD, UMR Innovation, Montpellier \\ ludovic.temple@cirad.fr \\ Guy FAURE \\ CIRAD, UMR Innovation, Montpellier \\ guy.faure@cirad.fr \\ Bernard TRIOMPHE \\ CIRAD, UMR Innovation, Montpellier \\ Bernard.triomphe@cirad.fr
}

Le concept de Système d'Innovation (SI) est présent depuis la fin des années 1980 dans un nombre important de recherches sur l'innovation, mais aussi de plus en plus dans les documents des politiques publiques d'innovation ou des instances internationales de développement (OCDE, UE, Banque Mondiale...). D'une manière générale, il vise à saisir comment un ensemble d'institutions, d'organisations, de réseaux et d'acteurs peuvent interagir pour favoriser l'innovation dans un espace donné national, régional ou sectoriel, ou dans un espace construit par des entreprises ou autour du développement d'une technologie (Carlsson et al., 2002). Employé à l'origine pour étudier les innovations technologiques dans l'industrie, puis le développement des " économies de la connaissance " (Foray, 2009), le concept s'est étendu à l'analyse des activités agricoles et agroalimentaires (World Bank, 2006). Il semble même avoir trouvé dans ce secteur un terrain propice, du fait de l'existence d'institutions spécialisées de recherche et de développement, et 
d'un renouvellement des enjeux de l'innovation agricole dans la perspective du développement durable (McIntyre et al., 2009). Des adaptations du concept de SI y sont proposées, comme celui d'" Agricultural Innovation System » (EU SCAR, 2012), mais ses définitions et usages apparaissent multiples, appelant à en faire une revue critique.

Cet article propose d'analyser comment différentes « communautés de connaissance » (Conein, 2004) utilisent le concept de SI dans l'agriculture ou l'agroalimentaire, et comment ces usages questionnent les spécificités de l'innovation dans ce secteur : les publications scientifiques de ces communautés témoignent-elles d'une simple application d'une approche générale des SI sur un secteur, ou donnent-elles lieu à des propositions plus originales, marquées par les conditions de l'innovation dans l'agriculture et l'agroalimentaire ? Pour répondre à ces questions, notre travail s'appuie sur une revue de la littérature et sur une étude bibliométrique réalisée à partir d'une sélection de revues internationales traitant de l'agriculture et de l'innovation.

Dans une première partie nous revenons sur l'évolution et la diversité des travaux mobilisant le concept de SI, pour proposer un cadre d'analyse autour de trois axes : le référentiel théorique et analytique, le domaine d'application, les finalités et usages du concept. Dans une deuxième partie, les résultats du travail bibliométrique sont présentés au regard d'indicateurs issus du cadre précédent. Ces résultats sont discutés dans une troisième partie, en suggérant l'existence de quatre communautés de connaissance qui interrogent différemment le caractère spécifique ou non des travaux mobilisant les SI pour étudier l'innovation agricole et/ou agroalimentaire.

\section{LE CONCEPT DE SYSTÈME D'INNOVATION : ANCRAGES THÉORIQUES, DOMAINES D'APPLICATION, USAGES}

\section{Origine et évolution du concept de système d'innovation}

Le concept de SI a été forgé par des auteurs qui ont examiné l'historique d'un certain nombre d'innovations et constaté que leur " réussite " pouvait être attribuée à l'existence d'institutions et de réseaux grâce auxquels les chercheurs et les entrepreneurs des secteurs public et privé ont collaboré, appris les uns des autres, partagé des ressources et agi pour faire face aux mutations des conditions économiques et techniques. Analysant sous cet angle le succès de l'économie japonaise dans les années 1970 et 1980, Freeman (1987) a été le premier à employer l'expression «système national d'innovation » pour désigner les institutions d'État associées à la définition 
et à l'application des politiques de recherche et d'innovation. Nelson (1993) et Lundvall (1992) ont ensuite étendu la définition de Freeman pour inclure l'ensemble des institutions et des acteurs industriels développant des activités de recherche et favorisant à l'échelle d'un pays la diffusion de connaissances pour l'innovation technologique.

À partir de ces travaux initiaux, les recherches utilisant la notion de SI se sont surtout développées autour des approches évolutionnistes de l'innovation, constituant progressivement les "Science Policy and Innovation Studies » (Martin, 2012). Le concept de SI est même retenu comme l'un des quatre piliers de cette communauté scientifique, dominée par les approches économiques, mais incluant aussi des chercheurs en sciences de gestion, histoire ou sociologie (Fagerberg, Verspagen, 2009). C'est dans ce cadre que s'est réalisée la majorité des déclinaisons du concept de SI (Edquist, 2004), dépassant les premières approches en termes de système national d'innovation : «systèmes régionaux d'innovation » rejoignant les travaux sur les clusters, districts ou milieux innovateurs (Cooke et al., 1998) ; « systèmes technologiques » ou « Corporate Innovation System » permettant de prendre en compte des interactions entre institutions et firmes au-delà de cadres politico-administratifs établis (Carlsson, 2006) ; systèmes sectoriels d'innovation analysant les conditions institutionnelles spécifiques à l'innovation dans un secteur d'activité (Malerba, 2002)... Parallèlement, les composantes des SI étaient précisées, intégrant des apports de communautés scientifiques proches (Carlsson et al., 2002) : prise en compte des mécanismes de diffusion de l'innovation ; caractérisation plus précise des institutions et de différentes « bases de connaissances »; analyse de réseaux d'entreprises ou d'acteurs... Des relations ou figures particulières ont aussi été mises en avant, comme le modèle de la "triple hélice » associant entreprises, universités et État (Leydesdorff, Etzkowitz, 1998).

D'autres courants de recherche ont cherché à intégrer ou adapter le concept de SI. Les travaux régulationnistes l'ont par exemple mobilisé dans l'analyse des transformations du capitalisme et de ses différentes formes nationales (Amable et al., 1997), y compris aux échelles territoriales et sectorielles (Lung, Bouneau, 2009). Les institutions dédiées à la recherche et à l'innovation trouvent alors leur place dans une analyse économique plus globale, où elles complètent les formes institutionnelles canoniques régulant le rapport de travail, la monnaie, la concurrence. Le concept de «système social d'innovation et de production » a été proposé pour prendre en compte les complémentarités institutionnelles jouant sur l'innovation et intégrer les dynamiques technologiques (Amable, 2003). Des sociologues participant au développement des « Science and Technology Studies » ont aussi mobilisé la notion de SI, mais sans l'adopter comme catégorie d'analyse à part entière. 
Elle a été ainsi utilisée pour décrire le contexte national du développement d'une technologie ou d'un réseau socio-technique (Naubahar, 2006 ; Hacket et al., 2008) ou pour situer les interactions d'acteurs clé comme les « intermédiaires de l'innovation » (Meyer, Kearnes, 2013). La référence aux SI est aussi présente dans les recherches en management de l'innovation (Smits, Kuhlman 2004 ; Shane 2008) ou dans les travaux sur la transition des systèmes socio-techniques qui revendiquent une filiation critique avec les approches évolutionnistes des Systèmes Sectoriel d'Innovation (Geels, 2004).

Influencées par ces travaux académiques, des agences de développement nationales et internationales se sont saisies du concept de SI. Depuis les années 1990, l’OCDE a élargi la définition du SI en y intégrant des " conditions-cadres » de l'innovation, telles que les régimes fiscaux, les réglementations, la culture et les comportements (OECD, 2001). La Banque Mondiale s'est aussi fait un relai pour médiatiser le concept de SI qu'elle définit comme " un réseau d'organisations, d'entreprises et d'individus focalisés sur l'exploitation économique de nouveaux produits, procédés et formes d'organisation, ainsi que les institutions et les politiques qui influencent leur comportement et leur performance » (World Bank, 2006). Elle a approfondi son engagement via la publication d'un compendium (Sourcebook) à destination des décideurs et investisseurs de la Banque et de ses partenaires, proposant un tour d'horizon du concept et de son application (World Bank, 2012). L'Union Européenne a aussi introduit le concept dans le cadre de sa stratégie globale vers une « économie à base de connaissance " (Borras, 2004). Les politiques nationales ou régionales d'innovation ont suivi ce mouvement, généralisant par exemple en France la notion de système régional d'innovation, en lui donnant un contenu normatif (Prager, 2010).

À partir des premières définitions, le concept de SI s'est donc enrichi et diversifié sous l'influence de plusieurs disciplines ou courants théoriques (dominés par la référence évolutionniste), mais aussi de confrontations à différents espaces d'application (pays, région, secteur, réseau d'entreprises...) avec différents usages (descriptif, analytique, normatif...). Les approches se répartissent entre une vision restreinte d'un SI, qui se limiterait aux institutions et réseaux dédiés à l'innovation (le contexte institutionnel et formel des processus d'innovation), et une vision plus large qui engloberait les structures informelles et les processus d'innovation et d'apprentissage eux-mêmes (Malerba, 2002). La diversité des définitions existantes de SI présente néanmoins un socle conceptuel commun, qui va au-delà des principes défendus par le noyau évolutionniste : une vision interactionniste de l'innovation, étendant l'analyse à une multiplicité d'acteurs et à l'environnement dans lequel ils opèrent ; un rôle central accordé aux connaissances et institutions, mais aussi (de manière plus ou moins affirmée) aux relations 
sociales et réseaux ; un cadre systémique cherchant à appréhender des régularités dans le réseau complexe d'acteurs et d'institutions à l'œuvre dans le processus d'innovation.

\section{Un cadre d'analyse pour les travaux se référant aux Systèmes d'Innovation}

Ce retour sur l'évolution des travaux sur les SI permet de proposer une grille d'analyse générique, dont le but est de préciser comment le concept est mobilisé dans des articles scientifiques, ouvrages ou documents politiques, en particulier dans un domaine comme l'agriculture et l'agroalimentaire. Cette grille d'analyse s'organise autour de trois axes, déjà suggérés par Amable (2003) ou Martin (2012), et plus largement par des recherches sur l'histoire des concepts en sciences sociales (Christin, 2010).

Le premier axe questionne le système de pensée, le paradigme ou cadre théorique auquel est associé (par le ou les auteurs) le concept de SI. Cette référence peut être explicite ou implicite. Le concept SI peut être restreint à une discipline scientifique ou à l'un de ses courants (par exemple évolutionniste ou institutionnaliste en économie), ou encore être promu comme catégorie interdisciplinaire. Mais au-delà des disciplines, il s'agit de préciser quels éléments et relations sont considérés pour définir le SI : acteurs individuels ou catégories d'acteurs, organisations, firmes, État, institutions, politiques publiques, réseaux, connaissances, système d'information, objets techniques, apprentissages, processus... Ce premier axe permet de clarifier l'approche et le paradigme de l'innovation associés au concept de SI, en s'interrogeant sur ses dimensions institutionnelle, cognitive, relationnelle et systémique. Il permet aussi de situer la vision du SI dans laquelle s'inscrit le texte : i) restreinte, formelle et souvent fonctionnaliste (les institutions dédiées à la recherche, l'enseignement et l'innovation), ii) élargie aux aspects informels du contexte social de l'innovation (réseaux, normes culturelles...), ou iii) encore plus large en incluant les processus d'innovation mis en ouvre dans les entreprises, c'est-à-dire un « système d'innovation et de production » (Malerba, 2002).

Le deuxième axe correspond au domaine et à l'espace d'application du concept, c'est-à-dire à la fois à la nature concrète des activités et des objets sur lesquels porte l'innovation ou auxquels le SI est dédié, et à l'extension du système lui-même : s'agit-il d'un espace politico-administratif dans lequel s'élaborent les institutions du SI (espace national, régional, sectoriel) ou d'un espace topologique construit à partir d'un ensemble d'entreprises (un groupe multinational ou un consortium) ou à partir de l'observation du processus 
d'innovation lui-même (système technologique, système socio-technique). Les travaux peuvent aussi se référer à plusieurs échelles et espaces, leur combinaison étant un enjeu majeur des réflexions actuelles sur l'innovation dans un contexte de globalisation de l'économie (Carlsson, 2006). La dimension temporelle est également un élément clé de la définition du domaine d'analyse : l'étude porte-t-elle sur un temps long (approche historique ou longitudinale) ou fait-elle référence à une période plus limitée (un état du SI) ?

Le troisième axe rend compte de l'usage social du concept, des finalités auxquelles il est associé, voire même des catégories d'acteurs ou classes sociales auxquelles il peut profiter. Le concept de SI est-il simplement utilisé pour décrire (ou évoquer) le contexte institutionnel d'une innovation étudiée? Vise-t-il au contraire à produire de nouvelles connaissances sur les institutions et réseaux de l'innovation, voire même sur les transformations plus globales d'un système économique et social ou l'évolution des relations entre sciences et société ? Le SI peut alors être considéré en soi comme objet de connaissance. Mais le concept peut aussi être directement instrumentalisé pour élaborer une politique de recherche ou d'innovation, réorganiser les relations entre les institutions de recherche et les acteurs économiques... Souvent associé à ces approches normatives, le SI peut également être utilisé pour évaluer les impacts des institutions de recherche sur le développement, en analysant leurs effets sur l'émergence et la diffusion d'innovations.

\section{MÉTHODE ET RÉSULTATS DE L'ÉTUDE BIBLIOMÉTRIQUE}

\section{Sélection des articles}

La grille d'analyse précédente nous permet d'explorer la production scientifique utilisant le concept SI dans des travaux sur l'agriculture et l'agroalimentaire. Nous avons pour cela réalisé une étude bibliométrique ${ }^{1}$ à partir de trois moteurs de recherche : $\mathrm{CAB}^{2}$, Web of Science ${ }^{3}$ et Scopus ${ }^{4}$. Ces bases ont été retenues car elles couvrent l'essentiel de la littérature internationale sur l'agriculture et l'agroalimentaire et se réfèrent à une diversité

1. Avec l'appui de Marie-Christine Duchamp du Cirad.

2. CAB est la base de données du Centre for Agriculture and Biosciences international : publications en agriculture et science de la vie depuis 1972: 7400 revues internationales, 7 millions de références.

3. Web of Science (WoS) est un module de la plateforme de l'Institute for Scientific Information. Multidisciplinaire, il recense plus de 10000 revues.

4. Scopus est une base de données de résumés et citations couvrant 19000 revues scientifiques. 
de disciplines scientifiques, même si certaines revues francophones ou de sciences humaines ne sont pas présentes. Les interrogations ont été faites sur les mots « Système d'Innovation » $(\mathrm{SI})$, puis « SI + Agro-alimentaire » ou «SI + Agriculture » ou «SI + biotechnologie » ou «SI + rural », respectivement en anglais, français, espagnol. La consultation a concerné le titre, le résumé et les mots-clés des articles, pour une période allant de 1995 à 2011.

Après un contrôle par lecture de l'ensemble des résumés, une base de 155 articles a été finalement retenue, incluant les articles concernant directement l'agriculture ou l'agroalimentaire, ainsi qu'une partie des articles sur les biotechnologies ou le développement rural, dès lors qu'ils apparaissaient liés à l'agriculture ou à l'agroalimentaire (par exemple semences OGM, biopesticides ou agrocarburants). Dans ce corpus, $59 \%$ des articles font en premier lieu référence à l'agriculture (comme secteur ou activité), $19 \%$ aux biotechnologies, $17 \%$ aux industries ou produits agroalimentaires et $5 \%$ à la gestion de ressources (eau, forêt, biodiversité) ou au développement rural (tableau 1).

Tableau 1 - Répartition des travaux sur Système d'Innovation suivant les domaines

\begin{tabular}{|l|c|c|}
\hline & Nombre d'articles & Fréquence \\
\hline Agriculture (production, conseil, recherche, secteur...) & 92 & $59 \%$ \\
\hline Biotechnologies (recherche, brevet, mise en production...) & 29 & $19 \%$ \\
\hline Agroalimentaire (transformation, produits...) & 26 & $17 \%$ \\
\hline Développement rural, gestion de ressources (eau, forêt...) & 7 & $5 \%$ \\
\hline Total Agriculture ou IAA + rural et biotechnologies liés & 155 & $100 \%$ \\
\hline
\end{tabular}

Quatre revues ont plus de 4 références et représentent ensemble près de $20 \%$ des articles : Research Policy, Agricultural Systems, Food Policy, Outlook on Agriculture... L'analyse chronologique des 155 articles (figure 1) souligne une forte augmentation de la production annuelle depuis 1996, avec trois périodes : une faible production de 1996 à 2000 (un ou deux articles par an) ; une croissance modérée entre 2001 et 2006 (entre 4 et 10 articles par an) ; une forte croissance de 2007 à 2011 (plus de 20 articles par an depuis 2009). 
Figure 1 - Évolution du nombre de publications faisant référence au concept SI et à l'agriculture ou l'agroalimentaire entre 1996 et $2011(\mathrm{~N}=155)$

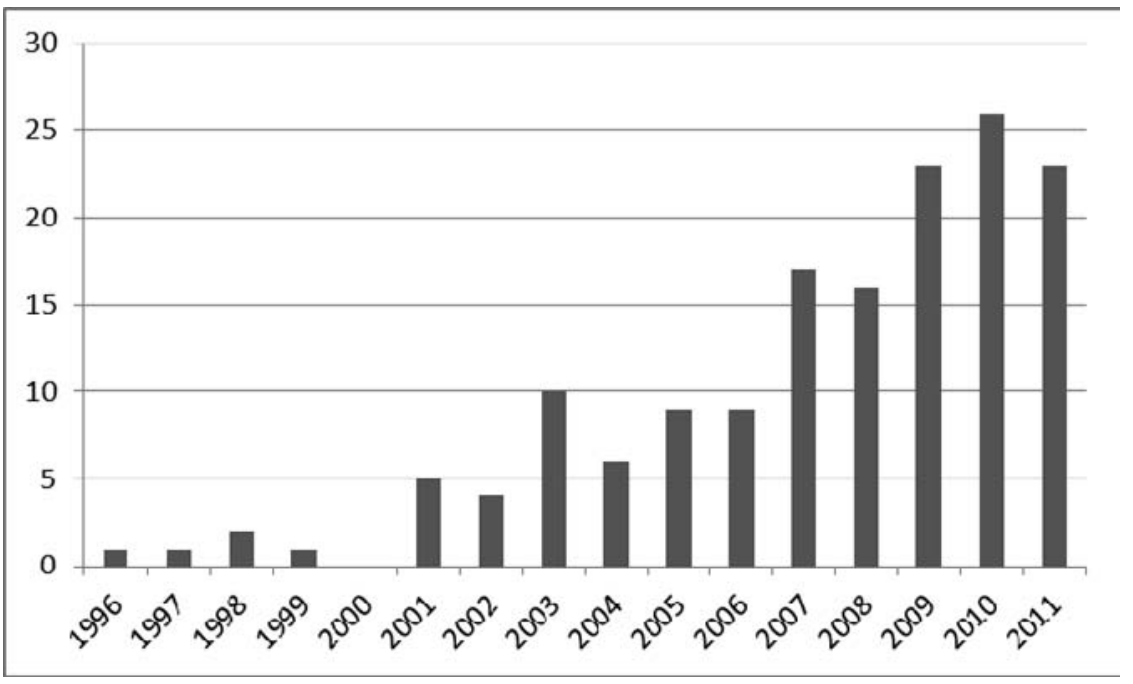

\section{Construction des variables et de leurs modalités}

Nous avons retenu deux ou trois variables par axe de notre grille d'analyse, et précisé leurs modalités (tableau2) :

- Deux variables indiquent le cadre théorique et analytique auquel se réfèrent les articles (premier axe) : un classement selon les disciplines et courants théoriques, lorsque cette référence est explicitée dans le résumé ou affichée clairement par les auteurs ; un recensement de l'occurrence de catégories analytiques dans le résumé (institution, connaissance, réseau...) ;

- Trois variables permettent de caractériser le domaine et l'extension des SI étudiés (deuxième axe) : l'activité principale (qui a permis de construire la base), la localisation des innovations ou SI (par catégories de pays) et l'échelle d'analyse (du local à l'international) ;

- Le décryptage des usages et finalités du concept SI dans chaque article (troisième axe) a été approché par deux variables : les références dans le résumé à une finalité, par exemple des recommandations politiques ou une évaluation d'impact ; la manière dont le concept SI est utilisé dans l'étude, celui-ci pouvant être l'objet de la recherche ou, à l'inverse, un simple élément de contexte. 
Tableau 2 - Variables et modalités retenues selon les 3 axes d'analyse des SI

\begin{tabular}{|c|c|}
\hline $\begin{array}{c}\text { Axe } \\
\text { d'analyse }\end{array}$ & Variables et leurs modalités retenues pour l'étude bibliométrique \\
\hline $\begin{array}{l}\text { Axe } 1 . \\
\text { Ancrage } \\
\text { théorique et } \\
\text { construction } \\
\text { du cadre } \\
\text { analytique }\end{array}$ & $\begin{array}{l}\text { 1.1. Référence théorique (expertise sur auteurs et références) : } \\
\text { évolutionniste, institutionnaliste, acteur-réseau/STS } \\
\text { économie agricole/sociologie rurale/farming system, sans référence } \\
\text { 1.2. Catégories analytiques retenues (citations dans résumé) : } \\
\text { institutions, networks, actors, knowledge, policy, research, food systems... }\end{array}$ \\
\hline $\begin{array}{l}\text { Axe } 2 . \\
\text { Domaine } \\
\text { d'application, } \\
\text { nature et } \\
\text { étendue des } \\
\text { phénomènes } \\
\text { étudiés }\end{array}$ & $\begin{array}{l}\text { 2.1. Activités ciblées par le SI (citations et expertise) : } \\
\text { Agriculture, biotechnologie, agroalimentaire, développement rural } \\
\text { 2.2. Localisation géographique de l'étude (citation et évaluation résumé) : } \\
\text { Pays moins avancés, émergents, en transition (Est et Moyen-Orient) } \\
\text { de l'OCDE anglophone, de l'OCDE méditerranéen, études comparatives } \\
\text { 2.3. Echelle du SI (citation résumé) : } \\
\text { national (SNII), régional SRI), local, international, sectoriel (SSI)... } \\
\text { espace topologique défini par des entreprises ou une technologie }\end{array}$ \\
\hline $\begin{array}{l}\text { Axe } 3 . \\
\text { Usage et } \\
\text { finalités du } \\
\text { concept }\end{array}$ & $\begin{array}{l}\text { 3.1. Finalités: } \\
\text { Orienter les politiques publiques, Évaluer l'impact de la recherche } \\
\text { Appuyer les entreprises, analyser une innovation ou les liens science/société } \\
\text { 3.2. Usage méthodologique du concept SI : } \\
\text { Aborder le SI comme objet global, étudier une composante d'un SI } \\
\text { Utiliser le SI comme facteur explicatif, contextualisation d'une étude }\end{array}$ \\
\hline
\end{tabular}

\section{Deux principaux référentiels théoriques mobilisés par les auteurs}

De manière générale le référentiel théorique est peu explicité dans les résumés, titres et mots clés. Un tiers des articles n'expriment pas de référentiel particulier (tableau 3). Ceux-ci correspondent généralement à des analyses descriptives, parfois écrits par des auteurs issus des sciences de l'ingénieur ou de disciplines techniques (agronomie, technologie agro-alimentaire). Parmi les autres articles, deux références théoriques dominent : i) Les travaux se référant à la sociologie et l'économie rurale, ou plus largement aux approches farming system, sont les plus nombreux (31\%). Ils concernent pour l'essentiel l'analyse d'innovations agricoles ou des évaluations du rôle de la recherche agronomique ; ii) le cadre évolutionniste de l'innovation (22\% des références) est repérable par l'usage de termes spécifiques (adoption, trajectoire technologique, capacité d'absorption, spillover...) et concerne surtout des revues comme Research Policy ou dédiées aux biotechnologies. Par ailleurs, des approches historiques ou institutionnalistes sont repérables mais moins présentes $(10 \%)$ et quelques articles $(5 \%)$ ont un caractère plus méthodologique (par exemple méthode d'analyse de réseau). 
Tableau 3 - Références disciplinaires des articles sur SI dans l'agriculture et l'agro-alimentaire

\begin{tabular}{|l|c|c|}
\hline & Nombre & Fréquence \\
\hline Sans référence théorique explicite & 51 & $33 \%$ \\
\hline Sociologie, économie rurale, Farming System & 48 & $31 \%$ \\
\hline Théorie évolutionniste, économie industrielle & 34 & $22 \%$ \\
\hline Approches institutionnalistes ou historiques & 15 & $10 \%$ \\
\hline Apport technique ou méthodologique & 7 & $5 \%$ \\
\hline Total & 155 & $100 \%$ \\
\hline
\end{tabular}

Le repérage des catégories d'analyse associées aux SI fait ressortir des notions générales comme « recherche » (présent dans 61 \% des résumés), « institution » $(45 \%)$ ou des concepts holistiques saisissant les relations entre activités (48 \%) : une filière produit (banane, manioc, vin..), un secteur ou sous-secteur (fruits, bioénergie...), une supply chain, un cluster... La citation de termes comme «politique » $(34 \%)$, « connaissance » $(32 \%)$ ou « acteurs » $(27 \%)$ suggère un socle analytique commun à de nombreux articles. Par contre seulement $14 \%$ des articles mentionnent le terme « réseau » dans leur résumé, indiquant que cette dimension n'est pleinement considérée que par une fraction des articles mobilisant le concept SI.

Tableau 4 - Occurrence des catégories dans les résumés des articles

\begin{tabular}{|l|c|c|}
\hline & Nombre d'articles & Fréquence \\
\hline Research & 96 & $62 \%$ \\
\hline (Agri)Food sector, chain ou system & 74 & $48 \%$ \\
\hline Institution/institutional & 69 & $45 \%$ \\
\hline Policy & 53 & $34 \%$ \\
\hline Knowledge & 49 & $32 \%$ \\
\hline Actor & 42 & $27 \%$ \\
\hline Networks & 22 & $14 \%$ \\
\hline
\end{tabular}

\section{Prévalence de trois blocs géographiques et des échelles nationales d'analyse}

Les travaux portent avant tout sur trois ensembles géographiques (tableau 5). Les Pays Moins Avancés (Afrique subsaharienne pour l'essentiel) sont les premiers concernés avec $34 \%$ des publications, privilégiant l'analyse d'innovations agricoles. Une raison est l'utilisation de l'approche SI par des chercheurs liés au CGIAR $^{5}$ ou à la Banque Mondiale pour proposer une restructuration de la recherche agronomique ou accélérer les transferts

5. Consultative Group on International Agronomic Research 
technologiques « nord-sud». Des chercheurs africains (Nigéria, Ghana, Afrique de l'est...) mobilisent aussi le concept SI pour analyser des innovations liées aux sociétés locales. Les pays anglophones de l'OCDE (principalement Canada, USA, Royaume Uni, Australie, pays nordiques) constituent le deuxième ensemble géographique ( $22 \%$ ), avec des thématiques focalisées sur les relations entre recherche publique et secteur privé, ou sur les biotechnologies (agrofourniture, création variétale, biocarburant). Les Pays émergents (Argentine, Brésil, Indonésie, Chine, Inde...) sont l'objet d'un nombre croissant d'articles (21\%), avec coexistence de travaux sur l'agriculture, l'agroalimentaire ou l'usage de biotechnologies et bioénergies. Les Pays en transition ou méditerranéens sont peu présents ( $6 \%$, avec des articles essentiellement sur l'Espagne), tout comme les articles comparatifs questionnant la dimension internationale des SI (moins de $15 \%$ des articles).

Tableau 5 - Répartition géographique des questions traitées par les articles sur SI

\begin{tabular}{|l|c|c|}
\hline & Nombre & Fréquence \\
\hline Pays Moins Avancés & 52 & $34 \%$ \\
\hline OCDE Anglophone & 34 & $22 \%$ \\
\hline Pays émergents & 33 & $21 \%$ \\
\hline OCDE Europe méditerranée & 9 & $6 \%$ \\
\hline Pays en transition & 7 & $5 \%$ \\
\hline International (comparaisons) & 20 & $13 \%$ \\
\hline Total & 155 & $100 \%$ \\
\hline
\end{tabular}

Les travaux se différencient aussi selon l'échelle d'analyse (tableau 6). Les échelles nationale ou sectorielle sont dominantes (plus de $60 \%$ des articles) à travers l'étude des rapports entre l'agriculture et le Système National d'Innovation ou celle d'un "Système d'Innovation Agricole », mais aussi en analysant l'innovation dans une filière nationale. Les Systèmes Régionaux d'Innovation (12\%) concernent avant tout les pays de l'OCDE. Les travaux qui étudient directement l'innovation à l'échelle mondiale et l'internationalisation des SI sont également peu nombreux (12 \%), même si cette question est souvent évoquée à travers le rôle de firmes multinationales ou des institutions internationales. Enfin, les articles abordant le SI à partir d'un réseau d'acteurs ou de firmes sont encore moins nombreux (10 \%) et assez hétérogènes, confirmant la prépondérance d'analyses très liées aux cadres politico-administratif pour l'agriculture. 
Tableau 6 - Echelle des Systèmes d'Innovation considérés dans les articles

\begin{tabular}{|l|c|c|}
\hline & Nombre & Fréquence \\
\hline Système National d'Innovation & 56 & $36 \%$ \\
\hline Système Sectoriel d'Innovation, AKIS ou AIS & 47 & $30 \%$ \\
\hline Système Régional d'Innovation & 18 & $12 \%$ \\
\hline Internationalisation des SI & 18 & $12 \%$ \\
\hline SI considéré à partir de réseaux de firmes & 16 & $10 \%$ \\
\hline Total & 155 & $100 \%$ \\
\hline
\end{tabular}

\section{Une orientation vers les politiques de recherche, d'innovation ou de développement}

La principale finalité de l'usage du SI dans l'agriculture (46\% des publications - tableau 7) concerne l'élaboration ou l'orientation des politiques publiques d'innovation, de recherche ou de développement. La référence est souvent explicite dans le résumé et le champ varie selon la nature de l'acteur public et son domaine d'intervention (transfert de connaissance, financement, promotion de biotechnologies...). L'évaluation de l'impact de la recherche est un objectif distinct, attribuable à $17 \%$ des articles. Les questions de méthode y sont très présentes, mais aussi la construction d'arguments visant à justifier les investissements de recherche. Les références à un appui direct aux entreprises sont par contre discrètes (8\%) et concernent par exemple le rôle de plateformes technologiques ou de «passeurs » assurant les liens entre public et privé. Enfin, près d'un tiers des articles ne fait pas référence à une finalité politique ou économique, mais affiche un objectif de production de connaissance sur l'innovation ou les institutions de recherche. Quelques travaux sont motivés par les débats sur le développement durable ou proposent un regard critique sur les modèles d'innovation.

Dans $22 \%$ des articles, le SI est un objet d'étude dans sa globalité, comme système. Plus souvent (42\%) le travail est centré sur une des composantes du SI (la recherche, les organisations de conseil, les relations entre firmes et institutions...). Moins d'un quart des articles ne mentionnent le SI que comme un facteur externe, influençant un processus d'innovation ou la stratégie d'une organisation. Enfin, dans $15 \%$ des articles le SI est évoqué comme simple contexte de l'étude. 
Tableau 7 - Usages et finalités du SI dans l'agriculture et l'agro-alimentaire

\begin{tabular}{|l|c|c|}
\hline & Nombre & Fréquence \\
\hline Orienter les politiques publiques & 72 & $46 \%$ \\
\hline Évaluer l'impact de la recherche & 27 & $17 \%$ \\
\hline Appuyer les entreprises et acteurs des filières & 12 & $8 \%$ \\
\hline Analyser une innovation, les liens recherche/société & 46 & $30 \%$ \\
\hline Aborder le SI comme objet global d'étude & 34 & $22 \%$ \\
\hline Étudier une composante d'un SI & 65 & $42 \%$ \\
\hline Utiliser le SI comme facteur explicatif & 34 & $22 \%$ \\
\hline Appréhender le SI comme un contexte de l'étude & 21 & $15 \%$ \\
\hline
\end{tabular}

\section{Le repérage de profils d'articles par l'analyse de correspondance multiple}

Pour synthétiser ces résultats et repérer les combinaisons entre ancrage théorique, domaine d'application et usages du SI, nous avons réalisé une analyse factorielle ${ }^{6}$ (Analyse des Correspondances Multiples) à partir du tableau de Burt rassemblant les variables qualitatives décrites précédemment. Les trois premiers axes factoriels ont un poids significatif ( $50 \%$ de l'inertie) et permettent de décrire les principales orientations des articles (figure 2).

Le premier axe factoriel (23\% de l'inertie) oppose (i) les articles qui portent sur l'agriculture, les PMA, se réfèrent à l'économie ou la sociologie rurales, sont ciblés sur une composante du SI et formulent des recommandations politiques... (ii) aux articles qui concernent les biotechnologies ou l'agroalimentaire, les pays de l'OCDE et mobilisent la référence évolutionniste ou la notion de Système Régional d'Innovation. On trouve ici une convergence entre les oppositions « Nord vs Sud », « technologies amont/ aval vs systèmes de production agricole », " approches évolutionniste vs issues de travaux plus ruralistes ».

Le deuxième axe factoriel (14\%) est marqué par la contribution des modalités « absence de référence théorique » et « usage du SI comme contexte ». Il oppose donc les articles ayant une référence limitée au concept de SI pour des études opérationnelles ou descriptives, à l'ensemble des autres usages qui analysent le SI dans sa globalité ou sur l'une de ses composantes, en se référant à un cadre théorique établi.

Le troisième axe factoriel (13\%) isole la contribution spécifique d'une référence historique ou institutionnaliste associée à l'absence de recommandation politique. Il oppose donc une posture critique et détachée de l'action, à

6. Avec l'appui de Salif Derra, doctorant Montpellier Supagro, Umr Innovation. 
des approches plus tournées vers les actions de développement ou politiques (se référant ou non aux autres cadres théoriques).

Figure 2 - Résultats de l'Analyse Factorielle (axe 1 et axe 2, axe 3 : + et -)

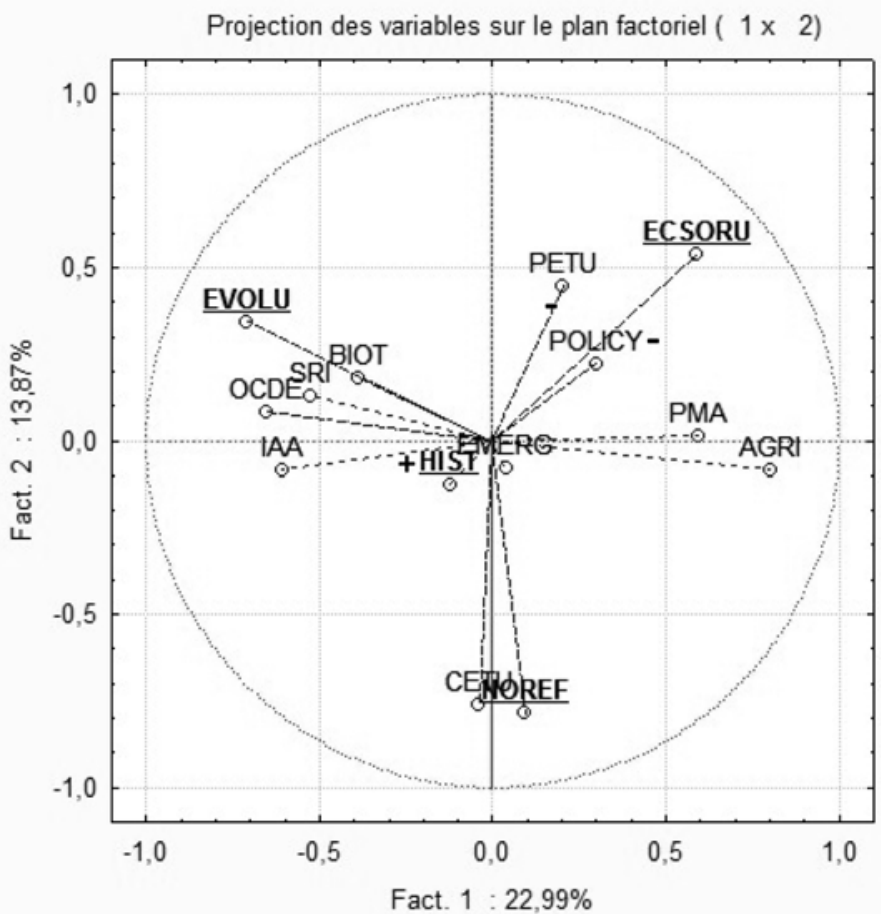

- Active

ECSORU : économie/sociologie rurale; EVOLU : évolutionniste ; NOREF : pas de référence ; HIST : Histoire AGRI : Agriculture ; BIOT : biotechnologies ; IAA : Agroalimentaire PMA : pays moins avancés ; OCDE : pays de l'OCDE; EMERG : pays émergents

CETU : contexte de l'étude ; PETU Partie du SI ; SRI : système régional d'Innovation ; POLICY : usage politique

Cette analyse factorielle montre le poids de la variable « cadre théorique » dont chaque modalité (à l'exception des travaux strictement méthodologiques) contribue fortement aux différents axes factoriels, et est liée à certaines modalités des autres variables. Une analyse des contingences entre les modalités de la référence théorique et celles des autres variables précise cette observation (tableau 8). 
Tableau 8 - Tableau de contingences entre références théoriques et 3 autres variables

\begin{tabular}{|l|c|c|c|c|c|c|c|c|c|c|c|c|}
\hline $\begin{array}{l}\text { Base } \\
\text { théorique }\end{array}$ & \multicolumn{3}{|c|}{ Domaine d'activité * } & \multicolumn{4}{c|}{ Pays** } & \multicolumn{5}{c|}{ Usage de la notion * } \\
\hline & AGRI & BIOT & IAA & RUR & PMA & EMER & OCDE & POLIC & GLOB & PETU & FACT & CETU \\
\hline $\begin{array}{l}\text { ECSORU } \\
48\end{array}$ & $42 * *$ & $3 *$ & $1 * *$ & 2 & $27 *$ & 7 & $7 *$ & $30 *$ & 10 & $28 *$ & 8 & $1 *$ \\
\hline $\begin{array}{l}\text { EVOLU } \\
34\end{array}$ & $4 * *$ & $15 *$ & $14 *$ & 1 & $3 * *$ & 8 & $16 *$ & 12 & $15 *$ & 10 & 7 & $1 *$ \\
\hline $\begin{array}{l}\text { HIST } \\
15\end{array}$ & 9 & 2 & 3 & 1 & $2 *$ & 4 & 5 & $1 *$ & 2 & 4 & $7 *$ & 2 \\
\hline $\begin{array}{l}\text { NOREF } \\
51\end{array}$ & 31 & 10 & 7 & 3 & 20 & 11 & 14 & 26 & $5 *$ & 17 & 12 & $17 *$ \\
\hline
\end{tabular}

148 articles (articles de méthode exclus); tests Chi2 **:p<0,01;*:0,01<p<0,05

ECSORU : économie/sociologie rurale ; EVOLU : évolutionniste ; HIST : Histoire ; NOREF : pas de référence AGRI : Agriculture ; BIOT : biotechnologies ; IAA : Agroalimentaire ; RUR : Rural

PMA : pays moins avancés ; EMER : pays émergents ; OCDE : pays de l'OCDE

GLOB : SI objet global d'étude; PETU partie du SI ; FACT : facteur explicatif ; CETU : contexte de l'étude

POLIC : usage politique

\section{COMMUNAUTÉS DE CONNAISSANCE ET SPÉCIFICITÉ AGRICOLE DES SI}

\section{Plusieurs communautés de connaissance mobilisent le concept de SI dans l'agriculture}

L'analyse bibliométrique suggère l'existence de 4 groupes d'articles marqués par des références théoriques différentes. Ces groupes peuvent être l'expression de communautés de connaissance (Wenger, 1998) impliquées dans la construction de différentes acceptions et usages du SI pour l'agriculture et l'agroalimentaire. Ces communautés sont des regroupements plus ou moins structurés de scientifiques utilisant la notion SI, associés à des acteurs politiques ou économiques. Elles peuvent être caractérisées selon les processus cognitifs ou des attributs qui les constituent, en se référant aux types avancés par les travaux en sociologie et économie de la connaissance (Cohendet et al., 2010) : communautés de "pratique » partageant des connaissances autour d'activités communes; communautés " épistémiques » qui s'organisent pour un projet cognitif commun ; communauté "d'intérêt » échangeant des informations du fait de positions, d'idées ou caractéristiques communes. Les résultats de notre analyse bibliométrique peuvent alors être interprétés en fonction de ces types et en mobilisant nos connaissances plus larges des recherches et acteurs du secteur agricole. Quatre communautés de connaissance sont suggérées, associées à différentes modalités du « cadre théorique » de l'analyse factorielle (figure 3). 
La première communauté associe des universitaires (en économie ou gestion) se référant globalement aux approches évolutionnistes de l'innovation, avec des acteurs des politiques de l'innovation (par exemple DG Recherche de l'UE), des organismes de développement et des firmes liées aux biotechnologies ou agroalimentaires, essentiellement dans les pays de l'OCDE ou émergents. Dans leurs articles, le concept de SI n'est généralement pas spécifié par rapport à l'agriculture. Par contre, sont mentionnées les catégories établies de SNI, SSI, SRI, clusters, ou le modèle «triple helice ». On retrouve dans cette communauté à la fois des travaux qui restent académiques et des études directement liées aux stratégies d'innovation nationales (Menrad, 2004) ou régionales (Asheim, Coenen, 2005), ou même à la promotion explicite de biocarburants et d'OGM par des firmes (Qaim, 2009). Les auteurs font référence à des processus d'innovation et au rôle des connaissances, mais les visions sont souvent assez proches de thèses diffusionnistes, accordant un rôle clé à la recherche et à l'évaluation des « conditions d'acceptabilité des innovations ", avec une attention croissante aux enjeux écologiques (Cunha et al., 2011). L'objet technologique (procédé de transformation alimentaire, biocarburant, OGM, NTIC appliquée à l'agriculture...) se prête bien à ce type d'analyse. On peut suggérer que dans ce groupe se construit plutôt une " communauté d'intérêt ", partageant une vision positive du progrès via les biotechnologies, entre des scientifiques trouvant une opportunité d'application d'un cadre théorique, des promoteurs de politiques de l'innovation et des firmes qui veulent instrumentaliser le concept pour favoriser les conditions de diffusion des technologies qu'elles produisent. L'attachement à cette vision technologique et économique du développement et la mobilisation d'un cadre théorique générique peuvent être associés à l'analyse d'une perte de spécificité du secteur agricole dans la globalisation : marchandisation et financiarisation, réindustrialisation de l'agriculture à partir des biotechnologies...

La deuxième communauté réunit des sociologues ou économistes du développement agricole ou rural (pays du sud et du nord), avec des responsables d'organisations de la recherche et du développement agricoles aux niveaux national ou international (CGIAR, DG agricole de l'UE...), et des agronomes impliqués dans des programmes de développement, surtout dans les PMA. Dans cette communauté sont mobilisés des concepts spécifiques aujourd'hui associés aux SI : Agricultural Knowledge and Innovation System (AKIS), Agricultural Innovation System (AIS) (Klerkx et al., 2010), Rural Innovation System (Spielman et al., 2010)... Les scientifiques viennent pour une large part d'une tradition de recherche constituée autour de l'agriculture (travaux sur le développement agricole, analyse des systèmes de recherche agronomiques, approches Farming System...) et sont associés à des institutions de recherche et de développement agronomique. Une pensée 
" réformiste » préoccupée par le développement durable est très présente (Coudel et al., 2012) et s'interroge sur la confrontation de processus top down (portés par les institutions) et bottom up (initiés par des agriculteurs et entrepreneurs ruraux) (Faure, Compagnone, 2011), sur la prise en compte de secteurs «traditionnel » et « entrepreneurial » (Adeoti, Olubamiwa, 2009), sur l'articulation de différentes formes de connaissances (Ekboir et al., 2008), ou sur la manière dont les institutions agricoles peuvent contribuer aux enjeux de réduction de la pauvreté, de sécurité alimentaire, de gestion de ressources naturelles (World Bank, 2006 ; Roling, 2009)... Des formes plus originales d'organisation des relations entre recherche et entreprises sont analysées ou suggérées, à l'image des plateformes régionales de concertation ou de « démarches participatives » (Abate et al., 2011). Cette communauté a davantage les caractéristiques d'une " communauté épistémique ", au moins en construction. Les articles scientifiques sont en effet complétés par des rapports, documents pédagogiques, appels à projets, avec une volonté d'organiser la promotion de nouvelles connaissances autour de l'approche en termes de SI dans l'agriculture (EU SCAR, 2012). Dans cette communauté, c'est la prise en compte de spécificités sectorielles qui justifie la constitution de catégories, d'approches et de concepts eux-mêmes spécifiques pour l'innovation et les SI.

La troisième communauté est constituée de scientifiques pouvant se référer au cadre évolutionniste ou à la sociologie rurale, mais qui témoigne surtout d'autres influences (histoire ou sociologie des sciences, économie institutionnelle, théorie de la régulation...). Ces travaux sont moins directement liés à des acteurs politiques ou économiques. Il s'agit d'études historiques, de synthèses, d'analyses comparées, de questionnements théoriques s'appuyant sur les transformations des activités agricoles et agroalimentaires (Allaire, Wolf 2004 ; Lindkvist, Sanchez 2008 ; Cypher, 2011). Le regard est plus critique, détaché des intérêts des acteurs économiques et politiques de l'agriculture. Ce groupe est plus restreint que les deux précédents et ne peut raisonnablement être associé à une communauté épistémique spécifique au secteur agricole, même si des références à la transition agroécologique ou à la diversité des trajectoires agroalimentaires pourraient y structurer un corpus de nouvelles connaissances (Touzard, 2009 ; Sanchez et al., 2010). À l'évidence ce n'est pas autour du concept de SI que se développe une approche critique de l'innovation dans l'agriculture. D'autres communautés scientifiques (Sociologie des Sciences et Technique notamment) ont pris cette place, par exemple autour de la revue Science, Technology and Human Values. Pour cette troisième communauté, la question de la spécificité agricole est plutôt posée comme question de recherche, à même d'opérer des comparaisons entre secteurs, mais elle ne justifie pas forcément la production de concepts et démarches spécifiques. 
La quatrième communauté est au contraire constituée à partir de scientifiques, d'ingénieurs et d'acteurs du secteur agricole, directement impliqués dans la mise en œuvre de processus d'innovation ou d'élaboration de politique agricole. Le concept de SI est utilisé pour contextualiser, analyser ou accompagner ces processus et pour pointer ses conditions institutionnelles, sans forcément interroger l'évolution ou les effets des institutions concernées. Les travaux francophones sur les SYAL commencent ainsi à s'y référer pour prendre en compte les contraintes et opportunités du contexte sectoriel ou national des innovations locales (Muchnik et al., 2007). L'usage du SI est plutôt descriptif ou rhétorique, et généralement pas central (contrairement à la deuxième communauté). Les scientifiques peuvent être associés à une ingénierie du développement ou de la formation qui se recentre sur les innovations en milieu rural et la recherche action (Sanginga et al., 2009 ; Faure et al., 2010). Derrière ce groupe peuvent en fait se repérer différentes communautés de pratiques, confrontées à la résolution de problèmes concrets, dans le cadre de réseaux différents (une entreprise et ses parties prenantes, un dispositif d'évaluation d'une politique publique, des projets de développement dans une région ou une filière). Dans ces communautés, la spécificité de l'usage du concept de SI est contrastée, répondant aux enjeux de résolution des problèmes rencontrés. Certains travaux se contentent d'une référence formelle aux propositions des communautés 1 et 2, d'autres sont amenés à « bricoler » les composantes d'un SI ou à adapter son usage.

Ces quatre communautés s'accordent globalement sur les principales caractéristiques des SI (innovation comme processus, rôle clé des institutions et connaissances, approche systémique) mais elles se distinguent par les catégories d'acteurs qui les composent (avec des positions et pratiques différentes au sein des SI), par les références théoriques et usages du concept de SI, et par une terminologie et un questionnement différents sur les spécificités agricoles et agroalimentaires. Elles sont connectées entre elles par certains auteurs clés (par exemple A Hall), mais elles sont aussi marquées par un dualisme important entre les communautés 1 et 2 : une opposition conceptuelle et méthodologique qui recouvre une opposition entre un modèle agroindustriel lié au développement des biotechnologies, et un modèle « alternatif » centré sur l'agriculture paysanne et l'agroécologie (Vanloqueren, Barret, 2009). 
Figure 3 - Les quatre communautés de connaissance sur Systèmes d'Innovation dans l'agriculture et l'agroalimentaire.

Schéma construit à partir de l'analyse factorielle

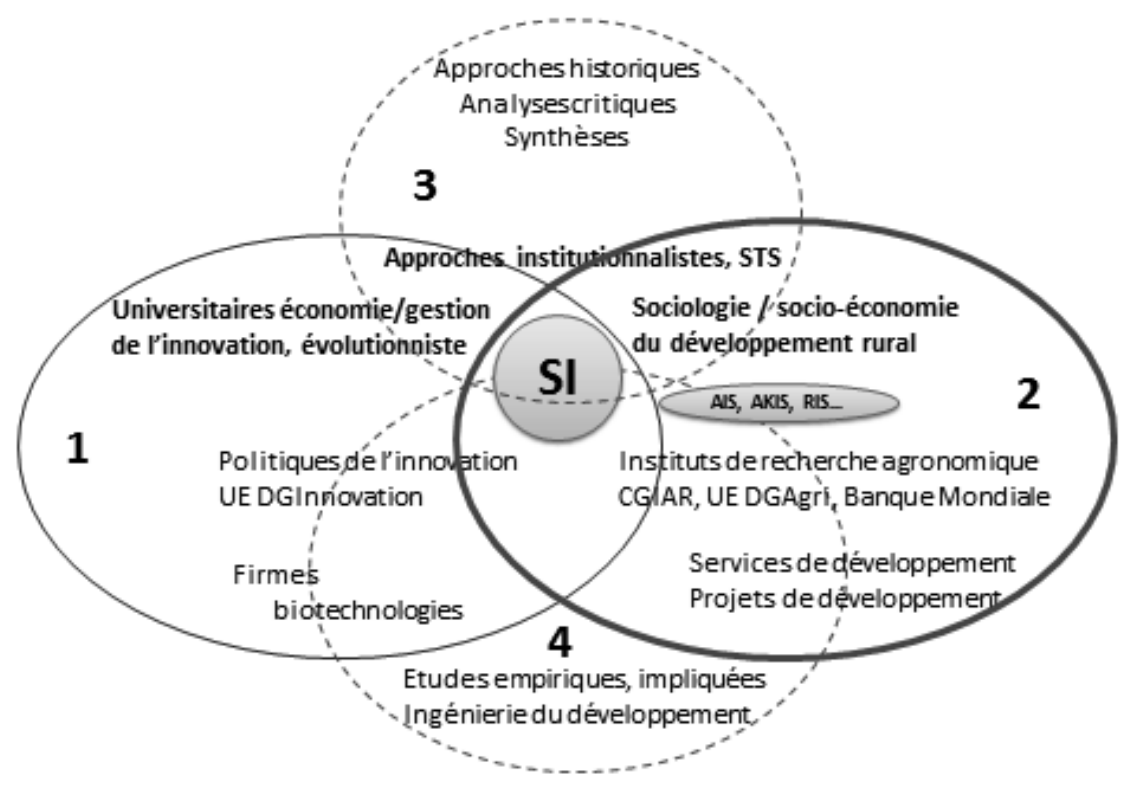

\section{Réinterroger la spécificité agricole de l'innovation,} des SI et des recherches sur les SI

Le repérage de ces communautés de connaissance amène alors à revenir sur les conditions d'une construction de définitions et d'usages du concept de SI qui seraient spécifiques à l'agriculture et l'agroalimentaire. Les quatre communautés repérées se distinguent en effet selon cette question. L'agriculture et l'agroalimentaire ne sont-ils qu'un domaine d'application d'un concept générique, ou bien ce secteur peut-il être une "niche scientifique » dans laquelle des questions et propositions nouvelles peuvent émerger du fait même de la confrontation aux réalités agricoles ? Ce questionnement peut être conduit en partant des arguments que développe la communauté épistémique qui cherche à produire des notions propres (AIS, AKIS...). Ces arguments partent d'un repérage des caractéristiques du secteur agricole qui peuvent induire des traits originaux dans ses processus d'innovation et dans ses SI :

- Des configurations d'acteurs et d'organisations particulières orientent l'innovation dans le secteur agricole et agroalimentaire. Les centres 
de recherche, de formation et de développement dédiés à l'agriculture font face à une atomisation d'exploitations agricoles, à une concentration des firmes d'amont et d'aval, mais aussi à l'existence d'acteurs spécifiques jouant le rôle de brokers (Klerkx, Leeuwis, 2009), comme des ONG, bureaux d'étude, syndicats... Ces configurations peuvent expliquer par exemple l'importance de l'action collective dans l'innovation agricole, le rôle que conservent des organisations publiques ou professionnelles assurant une économie d'échelle pour des activités de $R \& D$, les formes des réseaux d'innovation articulant liens locaux et à distances (Chiffoleau, Touzard, 2013 ; Spielman et al., 2010) ou encore des modes particuliers d'accompagnement ou de contrôle de l'innovation, mettant en tension des oligopoles privés, l'État, des organisations professionnelles et les consommateurs (contrôle des semences, biotechnologies, produits alimentaires ...).

- Plus largement ces acteurs s'insèrent dans des dispositifs institutionnels sectoriels constitués depuis longtemps, avec des politiques agricoles bien identifiées, mais aussi des formes particulières d'organisation de la production (travail familial, pluriactivité...), des échanges (circuits long vs courts, labels, Indications Géographiques...) et de la consommation (Temple et al., 2011). Ce cadre institutionnel associe des règles objectives et des compromis entre représentations sociales qui soutiennent une diversité de régimes d'innovation (Allaire, Wolf, 2004). L'exemple des indications géographiques, majoritairement liées aux produits agroalimentaires, illustre une forme de signalisation du produit qui s'appuie sur une codification des pratiques et des innovations, et suppose des actions collectives et des négociations politiques importantes pour modifier le cadre des innovations possibles.

- Au-delà de ces institutions, le secteur est marqué par une diversité de modèles ou systèmes agroalimentaires et leurs hybridations (Colonna et al., 2010). On peut même se demander si une spécificité du secteur agricole/agroalimentaire ne réside pas dans la coexistence d'une diversité de modèles agroalimentaires, parfois réduite à un dualisme entre un modèle « agroindustriel » aujourd'hui lié au développement des biotechnologies, et des modèles « alternatifs » voire traditionnels (dans les pays du Sud en particulier). L'innovation et les SI seraient alors à la fois inscrits dans chacun de ces systèmes agroalimentaires (dépendance de sentier), mais aussi dans leurs interactions économique et leurs confrontations politiques (Touzard, 2009).

- Plus fondamentalement peut-être, les rapports à la nature des activités et produits agricoles et agroalimentaires (systèmes biologiques, liens à la terre et aux écosystèmes, ingestion des aliments...) ont une 
influence sur l'innovation et les institutions qui les accompagnent. Ces rapports induisent à la fois des enjeux patrimoniaux, une forte inertie des investissements... et une forte instabilité liée à la saisonnalité, aux contraintes et considérations environnementales (dégradation et gestion des ressources naturelles), aux risques climatiques, à la périssabilité de nombreux produits ou aux enjeux sanitaires (Colonna et al., 2012). Ce contexte incertain joue sur la définition de domaines spécifiques d'intervention de la recherche et de l'innovation, mais aussi sur les conditions de mise en œuvre du changement technique. L'innovation apparaît à la fois plus risquée que dans d'autres secteurs et, de ce fait même, souvent confrontée à des formes apparentes de résistance ou d'inertie de la part des producteurs. Ces rapports à la nature peuvent être aussi considérés comme un des fondements de la dimension symbolique des aliments (Muchnik et al., 2007), jouant sur les domaines possibles ou interdits de l'innovation.

- De nombreux auteurs insistent plus largement sur les fonctions et les externalités de l'agriculture et par conséquence sur sa contribution (positive ou négative) à la production de biens publics : satisfaction de besoins alimentaires et impacts sur la santé, implication de l'activité dans la gestion de ressources naturelles, production de paysages et de biens culturels ou symboliques, occupation de l'espace... La reconnaissance et la gouvernance de ces biens publics justifient ainsi la combinaison d'actions collectives et d'interventions de l'État (Ostrom, 2011) et contribue fortement à publiciser les débats liés aux innovations dans l'agriculture (Coudel et al., 2010).

- La base de connaissance engagée dans les processus productif et innovants de l'agriculture et l'agroalimentaire est aussi pointée comme originale par différents travaux (Labarthe, 2005 ; Klerkx, Leuwis, 2009). Les multiples domaines d'apprentissage technique et organisationnel, la nécessité d'adaptation et d'expérimentation locale de connaissances génériques, l'importance de « connaissances tacites », mais aussi l'implication croissante des consommateurs citoyens dans les conditions de production orientent les besoins de formation et les formes de médiations associés à la construction de ces connaissances (Goulet, Vinck, 2012). La recherche elle-même, à travers ses expertises, est amenée à participer de manière nouvelle aux débats publics sur l'innovation alimentaire, exerçant, audelà de contributions classiques à l'élaboration d'une technologie, une fonction de légitimation du SI de l'agriculture et de l'agroalimentaire.

- Enfin, l'agriculture et l'agroalimentaire sont confrontés, sans doute plus que d'autres secteurs, à un renouvellement d'enjeux qui appellent à inscrire les innovations agricoles dans des perspectives de long terme : 
adaptations face au changement climatique (l'agriculture est le secteur économique le plus concerné), montée des questions de sécurité alimentaire, engagements à long terme dans des processus de gestion de la biodiversité et de ressources naturelles, révision structurelle du rôle de l'État (régionalisation) et redéfinition des rapports public/privé (Touzard, Temple, 2012). Ces enjeux plus complexes renforcent la nécessité d'inscrire l'innovation agricole dans des approches multidisciplinaires et prospectives (Coudel et al., 2012).

Au total, l'agriculture et l'agroalimentaire présentent des caractéristiques certes pas toujours exclusives à ce secteur, mais dont la combinaison offre un ensemble spécifique de conditions pour l'innovation et ses formes d'accompagnement, et donc pour les SI. Ces spécificités sectorielles peuvent justifier l'utilisation de démarches et concepts spécifiques, comme Agricultural Innovation System. Mais elles appellent aussi à développer les travaux sur les Systèmes Sectoriels d'Innovation (Malerba, 2002), en confrontant l'exemple agricole et agroalimentaire à d'autres secteurs pour renforcer l'édifice conceptuel permettant d'étudier l'innovation.

\section{CONCLUSION}

Nous avons montré en partant d'une analyse bibliométrique et bibliographique que le concept de SI connaît un développement important dans les travaux sur l'innovation dans l'agriculture et l'agroalimentaire. Le succès croissant du concept dans ce secteur apparaît lié à la co-évolution de plusieurs communautés de connaissance, cherchant pour certaines à appliquer le cadre théorique et analytique des Innovation Studies (Martin, 2012), pour d'autres à construire un corpus de concepts et méthodes plus original, dans la lignée de travaux de sociologie et d'économie rurale ou du développement. La question de la spécificité des conditions de l'innovation dans le secteur agricole est centrale et apparaît renouvelée du fait d'enjeux qui inscrivent aujourd'hui cette activité davantage dans les perspectives de long terme et dans les débats de la société. L'évolution de l'usage du concept dans les travaux sur l'innovation agricole n'est toutefois pas encore stabilisée. Du côté des travaux appliquant les concepts évolutionnistes, l'arbitrage entre la reconnaissance d'une spécificité sectorielle (via la notion de Système Sectoriel d'Innovation) ou la minimisation de cette question (accompagnant par exemple le développement des biotechnologies) n'est pas tranchée. D'un autre côté, la communauté épistémique issue de l'économie et la sociologie rurale, et liée à la réorientation des institutions de recherche agronomique et de développement agricole, hésite entre une inscription théorique plus 
claire dans les travaux promouvant les SI ou des notions proches (systèmes socio techniques), et la volonté de (re)construire un espace scientifique autonome et interdisciplinaire. Dans tous les cas, le maintien d'une dialectique entre plusieurs communautés de connaissance autour de l'usage de SI apparaît comme un gage de vivacité scientifique, à condition sans doute que les différentes communautés puissent interagir davantage.

\section{BIBLIOGRAPHIE}

ABATE, T., SHIFERAW, B., GEBEYEHU, S., AMSALU, B., NEGASH, K., ASSEFA, K., ESHETE, M., ALIYE, S., HAGMANN, J. (2011), A systems and partnership approach to agricultural research for development: Lessons from Ethiopia. Outlook on Agriculture, 40(3), 213-220.

ADEOTI, J. O., OLUBAMIWA, O. (2009), Towards an innovation system in the traditional sector: The case of the Nigerian cocoa industry. Science and Public Policy, 36(1), 15-31.

ALLAIRE, G. (1996). Emergence d'un nouveau système productif en agriculture, Canadian Journal of Agricultural Economics, 44(4), 461-479.

ALLAIRE, G., WOLF, S.A. (2004), Cognitive representations and institutional hybridity in agrofood innovation, Science Technology and Human Values, 29(4), 431-458.

AMABLE, B., BARRE, R., BOYER, R. (1997), Les systèmes d'innovation à l'ère de la globalisation, Economica, Paris.

AMABLE, B. (2003), Les Systèmes d'innovation, in Mustar, P., Penan, H. (dir.), Encyclopédie de l'innovation, Economica, Paris.

ASHEIM, B. T., COENEN, L. (2005), Knowledge bases and regional innovation systems: Comparing Nordic clusters, Research Policy, 34(8), 1173-1190.

BORRAS, S. (2004), System of innovation theory and the European Union, Science and Public Policy, 31(6), 425-433.

CARLSSON, B. (2006), Internationalization of innovation systems: A survey of the literature, Research Policy, 35, 56-67.

CARLSSON, B., JACOBSSON, S., HOLMÉN, M., RICKNE, A. (2002), Innovation systems: analytical and methodological issues, Research Policy, 31, 233-245.

CHIFFOLEAU, Y., TOUZARD, J.-M. (2013). Understanding Local Agri-Food Systems through advice network analysis, Agriculture and Human Values, online first

CHRISTIN, O. (2010), Dictionnaire des concepts nomades en Sciences Humaines, Paris, Métailié.

COHENDET, P., ROBERTS, J., SIMON, L. (2010). Les communautés de pratiques : une introduction, Revue Internationale de Gestion, 35, 31-35.

COLONNA, P., FOURNIER, S., TOUZARD, J.-M. (2011), Systèmes alimentaires, in Durabilité de l'alimentation face à de nouveaux enjeux, Esnouf, C., Russel, M., Bricas, N. (dir.), Versailles, QUAE, 59-84.

CONEIN, B. (2004), Communautés épistémiques et réseaux cognitifs, Revue d'Économie Politique, 113, 141-159.

COOKE, P., URANGA, M. G., ETXEBARRIA, G. (1998), Regional systems of innovation: an evolutionary perspective, Environment and Planning A, 30(9), 1563-1584. 
COUDEl, E., DEVAUTOUR, H., SOUlARD, T., FAURE, G., HUBERT, B. (2012), Apprendre à innover dans un monde incertain, Versailles, QUAE.

CUNHA, S. K., BOSZCZOWSKI, A. K., FACCO, C. A. (2011), Greening of sectoral innovation system in Brazil soybean, Agroalimentaria, 17(32), 71-86.

CYPHER, J. M. (2011), South America's Commodities boom: Developmental opportunity or path dependent reversion?, Canadian Journal of Development Studies, 30(3-4), 635-662.

EDQUIST, C. (2004), Systems of Innovation - A Critical Review of The State of the Art, in Fagerberg J., Mowery D., Nelson R. (eds), Handbook of Innovation, Oxford, Oxford University Press.

EKBOIR, J., SPIELMAN, D, DAVIS, K., OCHIENG, C. (2008), An innovation systems perspective on strengthening agricultural education and training in sub-Saharan Africa, Agricultural Systems, 98, 1-9.

EU SCAR (2012), Agricultural Knowledge and Innovation Systems in Transition, Brussels, European Commission.

FAGERBERG, J., VERSPAGEN, B. (2009), Innovation studies - the emerging structure of a new scientific field, Research Policy, 38, 218-233.

FAURE, G., GASSELIN, P., TRIOMPHE, B., TEMPLE, L., HOCDE, H. (2010), Innover avec les acteurs du monde rural : la recherche-action en partenariat, Versailles, QUAE.

FAURE, G., COMPAGNONE, C., (2011), Les transformations du conseil face à une nouvelle agriculture, Cahiers Agriculture, 20, 321-326.

FORAY, D. (2009). L'économie de la connaissance, Paris, La Découverte.

FREEMAN, C. (1987). Technology and Economic Performance: Lessons from Japan, London, Pinter.

FRYKFORS, C. O., JONSSON, H. (2010), Reframing the multilevel triple helix in a regional innovation system, Technology Analysis $\mathcal{E}$ Strategic Management, 22(7), 819-829.

GEELS, F. (2004), From sectoral Systems of innovation to socio-technical systems, Research Policy, 33, 897-920

GOULET, F., VINCK, D. (2012), L'innovation par retrait. Contribution à une sociologie du détachement, Revue Française de Sociologie, 53(2), 195-224.

HACKET, E., AMSTERDAMSKA, O., LYNCH, M., WAJCMAN, J. (2008), The Handbook Of Science and Technology Studies, Cambridge, The MIT Press.

HALL, A. (2005). Capacity development for agricultural biotechnology in developing countries: An innovation systems view of what it is and how to develop it, Journal of International Development, 17(5), 611-630.

KLERKX, L., LEEUWIS, C. (2009), Establishment and embedding of innovation brokers at different innovation system levels, Technological forecasting and Social Change, 76(6), 849-860.

KLERKX, L., AARTS, N., LEEUWIS, C. (2010). Adaptive management in agricultural innovation systems: The interactions between innovation networks and their environment, Agricultural Systems, 103(6), 390-400.

LABARTHE, P. (2005). Trajectoires d'innovation des services et inertie institutionnelle: dynamique du conseil dans trois agricultures européennes, Géographie, Économie et Société, 3(7), 289-311. 
LEVIDOW, L., BIRCH, K., PAPAIOANNOU, T. (2013), Divergent Paradigms of European Agro-Food Innovation The Knowledge-Based Bio-Economy (KBBE) as an R\&D Agenda, Science, Technology $\mathcal{B}$ Human Values, 38, 94-125.

LEYDESDORFF, L., ETZKOWITZ, H. (1998), The Triple Helix as a Model for Innovation Studies, Science Ë Public Policy, 25(3), 195-203.

LINDKVIST, K. B., SANCHEZ, J. L. (2008), Conventions and innovation: A comparison of two localized natural resource-based industries, Regional Studies, 42(3), 343-354.

LUNDVALL, B-A. (1992), National Innovation Systems: Towards a Theory of Innovation and Interactive Learning, London, Pinter.

LUNG, Y., BOUNEAU, C. (2009), Les dynamiques des systèmes d'innovation, Pessac, Editions de la MSHA.

MALERBA, F. (2002), The sectoral system of innovation and production, Research Policy, 31, 247-264.

MCINTYRE, B., HERREN, H., WAKHUNGU, J., WATSON, R. (2009), International Assessment of Agricultural Knowledge, Science and Technology for Development, Global report, FAO, World Bank.

MARTIN, B. (2012), The evolution of science policy and innovation studies, Research Policy, 41, 1219-1239.

MENRAD, K. (2004), Innovations in the food industry in Germany, Research Policy, 33(6-7), 845-878.

MEYER, M., KEARNES, M. (2013), Intermediaries between science, policy and the market, Science and Public Policy, 40(4), 423-429.

MUCHNIK, J., REQUIER-DESJARDINS, D., SAUTIER, D., TOUZARD, J.-M. (2007), Systèmes agroalimentaires localisées, Economies et Sociétés, Série AG, 29,1465-1484.

NAUBAHAR, S. (2006), Emergence and development of the National Innovation Systems concept, Research Policy, 35, 745-766.

NELSON, R. (1993), National Innovation Systems. A Comparative Analysis, New York/ Oxford, Oxford University Press.

OECD (2001), Innovative Clusters: Drivers of National Innovation Systems, Paris, OECD.

OSTROM, E. (2011), Par-delà les marchés et les Etats, Revue de l'OFCE, 120, 15-71.

PRAGER, J.-C. (2010), Méthode de diagnostic du système d'innovation dans les régions françaises, Ministère de l'Economie, de l'Industrie et de l'emploi, 125 p.

QAIM, M. (2009), Economics of Genetically Modified Crops, Annual Review of Resource Economics, 1, 665-693.

ROLING, N. (2009). Pathways for impact: scientists' different perspectives on agricultural innovation, International Journal of Agricultural Sustainability, 7(2), 83-94.

SANCHEZ, J. L., APARICIO, J., ALONSO, J. L. (2010), The shift between worlds of production as an innovative process in the wine industry in Castile and Leon, Geoforum, 41(3), 469-478.

SANGINGA, P, WATERS-BAYER, A, KAARIA, S, NJUKI, J, WETTASINHA, C. (2009), Innovation Africa: Enriching Farmers' Livelihoods, London, Earthscan.

SHANE, S. (2008), The Handbook of Technology and Innovation Management, London, Wiley. 
SMITS, R., KUHLMAN, S. (2004), The rise of systemic instrument of innovation policy, International Journal of Foresight and Innovation Policy, 1, 4-32.

SPIELMAN, D. J., DAVIS, K., NEGASH, M., AYELE, G. (2010), Rural innovation systems and networks: findings from a study of Ethiopian smallholders, Agriculture and Human Values, 1-18.

TEMPLE, L., LANÇON, F., PALPACUER, F., PACHE, G. (2011), Actualisation du concept de filière dans l'agriculture et l'agroalimentaire, Economies et sociétés, Série AG, 33, 1785-1797.

TOUZARD, J.-M. (2009), Quels apports de la Théorie de la Régulation à l'analyse des transformations agroalimentaires actuelles ?, Economies et Sociétés, Série AG, 31, 1923-1934.

TOUZARD, J.-M., TEMPLE, L. (2012), Sécurisation alimentaire et innovations dans l'agriculture et l'agroalimentaire : vers un nouvel agenda de recherche?, Cahiers Agriculture, $4(21)$.

VANLOQUEREN, G., BARET, P. V. (2009), How agricultural research systems shape a technological regime that develops genetic engineering but locks out agroecological innovations, Research Policy, 38(6), 971-983.

WENGER, E. (1998), Communities of Practice: Learning, Meaning, and Identity, Cambridge University Press.

WORLD BANK (2006), Enhancing Agricultural Innovation: How to Go Beyond the Strengthening of Research Systems, Washington, World Bank.

WORLD BANK (2012), Agricultural Innovation Systems. An Investment Sourcebook, Washington, World Bank. 